TRANSACTIONS OF THE

AMERICAN MATHEMATICAL SOCIETY

Volume 359, Number 8, August 2007, Pages 4055-4072

S 0002-9947(06)04296-6

Article electronically published on August 16, 2006

\title{
MINIMAL POLYNOMIALS AND RADII OF ELEMENTS IN FINITE-DIMENSIONAL POWER-ASSOCIATIVE ALGEBRAS
}

\author{
MOSHE GOLDBERG
}

\begin{abstract}
In the first section of this paper we revisit the definition and some of the properties of the minimal polynomial of an element of a finitedimensional power-associative algebra $\mathcal{A}$ over an arbitrary field $\mathbb{F}$. Our main observation is that $p_{a}$, the minimal polynomial of $a \in \mathcal{A}$, may depend not only on $a$, but also on the underlying algebra. More precisely, if $\mathcal{A}$ is a subalgebra of $\mathcal{B}$, and if $q_{a}$ is the minimal polynomial of $a$ in $\mathcal{B}$, then $p_{a}$ may differ from $q_{a}$, in which case we have $q_{a}(t)=t p_{a}(t)$.

In the second section we restrict attention to the case where $\mathbb{F}$ is either the real or the complex numbers, and define $r(a)$, the radius of an element $a$ in $\mathcal{A}$, to be the largest root in absolute value of the minimal polynomial of $a$. We show that $r$ possesses some of the familiar properties of the classical spectral radius. In particular, we prove that $r$ is a continuous function on $\mathcal{A}$.

In the third and last section, we deal with stability of subnorms acting on subsets of finite-dimensional power-associative algebras. Following a brief survey, we enhance our understanding of the subject with the help of our findings of the previous section. Our main new result states that if $\mathcal{S}$, a subset of an algebra $\mathcal{A}$, satisfies certain assumptions, and $f$ is a continuous subnorm on $\mathcal{S}$, then $f$ is stable on $\mathcal{S}$ if and only if $f$ majorizes the radius $r$ defined above.
\end{abstract}

\section{The Minimal POLYNOMial REVISITED}

Let $\mathcal{A}$ be a nontrivial, finite-dimensional algebra over a field $\mathbb{F}$. Throughout this paper we assume that $\mathcal{A}$ is power-associative; i.e., that the subalgebra generated by any one element of $\mathcal{A}$ is associative, thus ensuring that powers of each element in $\mathcal{A}$ are uniquely defined.

Let $a$ be an element of $\mathcal{A}$. As usual, by a minimal polynomial of $a$ we mean a monic polynomial of lowest positive degree with coefficients in $\mathbb{F}$ that annihilates a.

Surely, if $\mathcal{A}$ does not have a unit, and $p$ is a nonzero member of $\mathbb{F}[t]$, the ring of polynomials over $\mathbb{F}$, then an element $a$ of $\mathcal{A}$ can be substituted in $p$ only if $p$ does not have a constant term; so in this case, a minimal polynomial of $a$ must be void of a constant term. With this in mind, we record the following familiar assertion.

Received by the editors December 18, 2005 and, in revised form, April 17, 2006.

2000 Mathematics Subject Classification. Primary 15A60, 16B99, 17A05, 17A15.

Key words and phrases. Finite-dimensional power-associative algebras, minimal polynomial, radius of an element in a finite-dimensional power-associative algebra, norms, subnorms, submoduli, stable subnorms.

(C)2006 American Mathematical Society Reverts to public domain 28 years from publication 
Theorem 1.1. Let $\mathcal{A}$ be a finite-dimensional power-associative algebra over $\mathbb{F}$. Then:

(a) Every element $a \in \mathcal{A}$ possesses a unique minimal polynomial.

(b) The minimal polynomial of a divides every other polynomial in $\mathbb{F}[t]$ that annihilates a.

Proof. For completeness, we first address the often encountered case where $\mathcal{A}$ has a unit, say $e$. Let $\operatorname{dim} \mathcal{A}=m$, and select $a \in \mathcal{A}$. Then the elements

$$
e, a, \ldots, a^{m}
$$

are linearly dependent in $\mathcal{A}$. So $\mathcal{K}$, the set of polynomials in $\mathbb{F}[t]$ that annihilates $a$, contains a nonzero polynomial. Further, it is easily verified that $\mathcal{K}$ is an ideal (and hence a principal ideal) in $\mathbb{F}[t]$. Thus, (e.g., $\mathbb{B M}$, Section 3.8, Theorem 11], $\underline{\mathbb{R}}$, Theorem 3.54]), $\mathcal{K}$ is generated by a unique monic polynomial of positive degree, and the proof follows.

If $\mathcal{A}$ does not have a unit, the above argument must be slightly modified: Selecting $a \in \mathcal{A}$, we note that the elements

$$
a, \ldots, a^{m+1}
$$

are linearly dependent. So $\tilde{\mathcal{K}}$, the set of polynomials in $\mathbb{F}[t]$ that have no constant term and annihilate $a$, contains a nonzero member. Now, take $p$ in $\tilde{\mathcal{K}}$ and let

$$
q(t)=\alpha_{n} t^{n}+\cdots+\alpha_{1} t+\alpha_{0}
$$

be an arbitrary polynomial in $\mathbb{F}[t]$. Then,

$$
\begin{aligned}
(p q)(t) & =p(t)\left(\alpha_{n} t^{n}+\cdots+\alpha_{1} t+\alpha_{0}\right) \\
& =\alpha_{n} p(t) t^{n}+\cdots+\alpha_{1} p(t) t+\alpha_{0} p(t) .
\end{aligned}
$$

Hence it is permissible to substitute $a$ in $p q$, which gives

$$
(p q)(a)=\alpha_{n} p(a) a^{n}+\cdots+\alpha_{1} p(a) a+\alpha_{0} p(a) .
$$

Since $p(a)=0$, it follows that $(p q)(a)=0$. So $\tilde{\mathcal{K}}$ is an ideal in $\mathbb{F}[t]$, and the rest of the proof follows just as in the previous case.

As we shall see in the following two examples, $p_{a}$, the minimal polynomial of an element $a \in \mathcal{A}$, may depend not only on $a$, but also on the underlying algebra. Thus, from now on we shall often refer to $p_{a}$ as the minimal polynomial of a in $\mathcal{A}$.

Example 1.1. Consider the matrix algebras

$$
\mathcal{A}=\left\{\left(\begin{array}{ccc}
0 & 0 & 0 \\
0 & \alpha_{22} & \alpha_{23} \\
0 & \alpha_{32} & \alpha_{33}
\end{array}\right): \alpha_{j k} \in \mathbb{F}\right\}
$$

and

$$
\mathcal{B}=\left\{\left(\begin{array}{ccc}
0 & 0 & 0 \\
\alpha_{21} & \alpha_{22} & \alpha_{23} \\
\alpha_{31} & \alpha_{32} & \alpha_{33}
\end{array}\right): \alpha_{j k} \in \mathbb{F}\right\},
$$

with the usual matrix operations. Evidently, $\mathcal{A}$ is a subalgebra of $\mathcal{B}$. Further, $\mathcal{A}$ has a unit,

$$
I_{\mathcal{A}}=\left(\begin{array}{ccc}
0 & 0 & 0 \\
0 & 1 & 0 \\
0 & 0 & 1
\end{array}\right)
$$


whose minimal polynomial in $\mathcal{A}$ is $p_{I_{\mathcal{A}}}(t)=t-1$. The algebra $\mathcal{B}$, on the other hand, does not have a unit; for if it did, say $I_{\mathcal{B}}$, then multiplying $I_{\mathcal{B}}$ from the left by $E_{31}$, the $3 \times 3$ matrix all of whose entries are zero except for the lower-left entry which is 1 , we would get $E_{31}=E_{31} I_{\mathcal{B}}=0$, a contradiction. It thus follows that $p_{I_{\mathcal{A}}}$, having a constant term, is not the minimal polynomial of $I_{\mathcal{A}}$ in $\mathcal{B}$. Indeed, it is not hard to verify that $q_{I_{\mathcal{A}}}$, the minimal polynomial of $I_{\mathcal{A}}$ in $\mathcal{B}$, is

$$
q_{I_{\mathcal{A}}}(t)=t p_{I_{\mathcal{A}}}(t)=t^{2}-t
$$

We observe that for other elements of $\mathcal{A}$, the minimal polynomials in $\mathcal{A}$ and in $\mathcal{B}$ may coincide; for instance, the minimal polynomials of

$$
A=\left(\begin{array}{lll}
0 & 0 & 0 \\
0 & 1 & 1 \\
0 & 1 & 1
\end{array}\right)
$$

in $\mathcal{A}$ and in $\mathcal{B}$ are both equal to $t^{2}-2 t$.

Example 1.2. Let $\mathcal{A}$ be the algebra in (1.1), and take $\mathcal{B}$ to be $\mathbb{F}^{3 \times 3}$, the algebra of $3 \times 3$ matrices over $\mathbb{F}$ with the usual operations. Then, in contrast to Example 1.1, both $\mathcal{A}$ and $\mathcal{B}$ have (different) units. Yet, just as in Example 1.1, the minimal polynomials of $I_{\mathcal{A}}$ in $\mathcal{A}$ and in $\mathcal{B}$ are $p_{I_{\mathcal{A}}}(t)=t-1$ and $q_{I_{\mathcal{A}}}(t)=t p_{I_{\mathcal{A}}}(t)=t^{2}-t$ respectively, and those of $A$ satisfy $p_{A}(t)=q_{A}(t)=t^{2}-2 t$.

As we shall now see, Examples 1.1 and 1.2 are but special cases of the following more general phenomenon.

Theorem 1.2. Let $\mathcal{A}$ and $\mathcal{B}$ be finite-dimensional power-associative algebras over $\mathbb{F}$, such that $\mathcal{A}$ is a subalgebra of $\mathcal{B}$. Let a be an element of $\mathcal{A}$, and let $p_{a}$ and $q_{a}$ denote the minimal polynomials of a in $\mathcal{A}$ and in $\mathcal{B}$, respectively. Then either $p_{a}=q_{a}$ or $q_{a}(t)=t p_{a}(t)$. More specifically:

(a) If either $\mathcal{A}$ and $\mathcal{B}$ do not have units, or $\mathcal{A}$ and $\mathcal{B}$ have the same unit, or $\mathcal{A}$ does not have a unit but $\mathcal{B}$ does, then $p_{a}=q_{a}$.

(b) If either $\mathcal{A}$ has a unit but $\mathcal{B}$ does not, or $\mathcal{A}$ and $\mathcal{B}$ have different units, then $p_{a}=q_{a}$ or $q_{a}(t)=t p_{a}(t)$.

Proof. If either $\mathcal{A}$ and $\mathcal{B}$ do not have units, or $\mathcal{A}$ and $\mathcal{B}$ have the same unit, then the proof that $p_{a}=q_{a}$ is straightforward and is left to the reader.

To complete the proof of (a), assume that $\mathcal{A}$ does not have a unit but $\mathcal{B}$ does. Note that if $q_{a}$ has no constant term, then again, $p_{a}=q_{a}$. Otherwise, $q_{a}$ is of the form

hence,

$$
q_{a}(t)=\beta_{n} t^{n}+\cdots+\beta_{1} t+\beta_{0}, \quad \beta_{0} \neq 0
$$

$$
q_{a}(a)=\beta_{n} a^{n}+\cdots+\beta_{1} a+\beta_{0} e_{\mathcal{B}}=0,
$$

where $e_{\mathcal{B}}$ is the unit in $\mathcal{B}$. Consequently,

$$
e_{\mathcal{B}}=-\beta_{0}^{-1}\left(\beta_{n} a^{n}+\cdots+\beta_{1} a\right) \in \mathcal{A}
$$

so $e_{\mathcal{B}}$ is a unit in $\mathcal{A}$, a contradiction.

As for the proof of (b), we attend first to the case where $\mathcal{A}$ has a unit but $\mathcal{B}$ does not. Let

$$
p_{a}(t)=\alpha_{m} t^{m}+\cdots+\alpha_{1} t+\alpha_{0}
$$

be the minimal polynomial of $a$ in $\mathcal{A}$. If $\alpha_{0}=0$, then once again, $p_{a}=q_{a}$. So suppose $\alpha_{0} \neq 0$, in which case $q_{a}$, having no constant term, must differ from $p_{a}$. 
Consider the monic polynomial $q(t)=t p_{a}(t)$ which has no constant term and annihilates $a$. If $q=q_{a}$, then we are done. Otherwise, $q$ is not the minimal polynomial of $a$ in $\mathcal{B}$; so $\operatorname{deg} q_{a}<\operatorname{deg} q$. Consequently, $\operatorname{deg} q_{a} \leq \operatorname{deg} p_{a}$, and since $p_{a} \neq q_{a}$, it follows that $p_{a}$ is not the minimal polynomial of $a$ in $\mathcal{A}$, a contradiction.

Finally, assume that $\mathcal{A}$ and $\mathcal{B}$ have different units. As before, let $\alpha_{0}$ and $\beta_{0}$ denote the constant terms of $p_{a}$ and $q_{a}$, respectively. If $\alpha_{0}$ and $\beta_{0}$ are both zero, then once more we conclude that $p_{a}=q_{a}$. Further, if $\beta_{0} \neq 0$, then by (1.2), $e_{\mathcal{B}}$ is a unit in $\mathcal{A}$, a contradiction. Hence, it remains to examine the case where $\alpha_{0} \neq 0$ and $\beta_{0}=0$. Consider again the monic polynomial $q(t)=t p_{a}(t)$. If $q=q_{a}$, then there is nothing left to be proved. If not, then as in the previous case, $\operatorname{deg} q_{a}<\operatorname{deg} q$; hence, $\operatorname{deg} q_{a} \leq \operatorname{deg} p_{a}$ and it follows that $p_{a}$ is not the minimal polynomial of $a$ in $\mathcal{A}$, a contradiction.

The two cases in part (b) of Theorem 1.2 are illustrated by Examples 1.1 and 1.2 above. Regarding part (a) of the theorem, we give two additional examples of a more general flavor.

Example 1.3. Let $a$ be an element of a finite-dimensional power-associative algebra $\mathcal{A}$ with a unit, and denote by $\mathcal{A}_{a}$ the associative (even commutative) subalgebra of $\mathcal{A}$ generated by $a$ and by the unit. If $b$ is an element of $\mathcal{A}_{a}$, then by the first case in part (a) of Theorem 1.2, the minimal polynomials of $b$ in $\mathcal{A}$ and in $\mathcal{A}_{a}$ coincide.

Example 1.4. Suppose $\mathcal{A}$ does not have a unit, and let $\mathcal{B}$ be the algebra obtained by adding a unit to $\mathcal{A}$ in the familiar manner; that is,

$$
\mathcal{B}=\mathbb{F} \oplus \mathcal{A},
$$

where scalar multiplication and addition are defined componentwise, and multiplication is given by

$$
(\alpha+a)(\beta+b)=\alpha \beta+(a b+\beta a+\alpha b), \quad a, b \in \mathcal{A}, \quad \alpha, \beta \in \mathbb{F} .
$$

Clearly, $e=1+0$ is a unit in $\mathcal{B}$, and $\mathcal{A}$ is a subalgebra (even an ideal) in $\mathcal{B}$. Thus, by the third case in part (a) of Theorem 1.2, if $a \in \mathcal{A}$, then the minimal polynomials of $a$ in $\mathcal{A}$ and in $\mathcal{B}$ are identical. Note that since $\mathcal{A}$ is power-associative, so is $\mathcal{B}$. Similarly, if $\mathcal{A}$ happens to be associative, then $\mathcal{B}$ is associative too.

For our next example we shall need the following assertion that hardly requires a proof.

Proposition 1.1. Let $\mathcal{A}$ and $\mathcal{B}$ be algebraically isomorphic, finite-dimensional, power-associative algebras over $\mathbb{F}$, with an algebra isomorphism $\varphi: \mathcal{A} \rightarrow \mathcal{B}$. Let a be an element of $\mathcal{A}$, and let $p_{a}$ and $q_{\varphi(a)}$ denote the minimal polynomials of a in $\mathcal{A}$ and of $\varphi(a)$ in $\mathcal{B}$, respectively. Then $p_{a}=q_{\varphi(a)}$.

Example 1.5. Let $\mathcal{A}$ be an $m$-dimensional, associative algebra with a unit $e$. Consider the regular left representation which associates with each element $a$ of $\mathcal{A}$ the linear transformation $T_{a}: \mathcal{A} \rightarrow \mathcal{A}$, defined by

$$
T_{a}(x)=a x, \quad x \in \mathcal{A} .
$$

Let $\mathbb{F}^{m \times m}$ denote the algebra of $m \times m$ matrices over $\mathbb{F}$ with the usual operations. Select a basis $\left\{b_{1}, \ldots, b_{m}\right\}$ of $\mathcal{A}$, and for each element $a \in \mathcal{A}$ let $A_{a}$ be the matrix in $\mathbb{F}^{m \times m}$ that represents $T_{a}$ with respect to this basis. Then obviously, the familiar mapping $\varphi: \mathcal{A} \rightarrow \mathbb{F}^{m \times m}$, defined by

$$
\varphi(a)=A_{a}, \quad a \in \mathcal{A},
$$


satisfies

$$
\varphi(\alpha a+\beta b)=\alpha \varphi(a)+\beta \varphi(b), \quad \varphi(a b)=\varphi(a) \varphi(b), \quad a, b \in \mathcal{A}, \quad \alpha, \beta \in \mathbb{F} .
$$

Further, $\operatorname{Ker} \varphi=\{0\}$ since $a \in \operatorname{Ker} \varphi$ implies $a=a e=0$. Hence $\mathcal{A}$ is algebraically isomorphic to $\mathcal{A}_{\varphi}=\left\{A_{a}: a \in \mathcal{A}\right\}$, the image of $\mathcal{A}$ under $\varphi$; so by Proposition 1.1, the minimal polynomials of $a$ in $\mathcal{A}$ and of $A_{a}$ in $\mathcal{A}_{\varphi}$ coincide. Moreover, since $T_{e}$ is the identity transformation on $\mathcal{A}$, we observe that regardless of the choice of our basis, $\varphi(e)$, the unit in $\mathcal{A}_{\varphi}$, is $I_{m}$, the identity in $\mathbb{F}^{m \times m}$. So, by Theorem 1.2(a), the minimal polynomial of $A_{a}$ in $\mathcal{A}_{\varphi}$ is that of $A_{a}$ in $\mathbb{F}^{m \times m}$. Thus, the minimal polynomials of $a$ in $\mathcal{A}$ and of $A_{a}$ in $\mathbb{F}^{m \times m}$ are one and the same; so in particular, the roots of the minimal polynomial of $a$ in $\mathcal{A}$ are the eigenvalues of $A_{a}$.

If $\mathcal{A}$ is an $m$-dimensional associative algebra without a unit, we modify the above discussion by adjoining a unit to $\mathcal{A}$, obtaining, as in Example 1.4, the $(m+$ 1)-dimensional associative algebra $\mathcal{B}=\mathbb{F} \oplus \mathcal{A}$. Selecting a basis $\left\{b_{1}, \ldots, b_{m+1}\right\}$ for $\mathcal{B}$, we now let $\psi: \mathcal{B} \rightarrow \mathbb{F}^{(m+1) \times(m+1)}$ be the mapping that associates with each element $a$ in $\mathcal{B}$ the matrix $A_{a}$ which represents the regular left representation $T_{a}: \mathcal{B} \rightarrow \mathcal{B}$ with respect to this basis. Hence, by our previous discussion, $\mathcal{B}$ is algebraically isomorphic to the $(m+1)$-dimensional subalgebra $\mathcal{B}_{\psi}=\left\{A_{a}: a \in\right.$ $\mathcal{B}\}$ of $\mathbb{F}^{(m+1) \times(m+1)}$; and consequently, our original algebra $\mathcal{A}$ is algebraically isomorphic to the $m$-dimensional subalgebra $\mathcal{A}_{\psi}=\left\{A_{a}: a \in \mathcal{A}\right\}$ of $\mathcal{B}_{\psi}$, the image of $\mathcal{A}$ under the mapping

$$
\psi(a)=A_{a}, \quad a \in \mathcal{A} .
$$

Now, by Proposition 1.1, the minimal polynomials of $a$ in $\mathcal{A}$ and of $A_{a}$ in $\mathcal{A}_{\psi}$ coincide. Also, since $\mathcal{A}$ has no unit, $\mathcal{A}_{\psi}$ has no unit; so by Theorem 1.2(a), the minimal polynomial of $A_{a}$ in $\mathcal{A}_{\psi}$ is that of $A_{a}$ in $\mathbb{F}^{(m+1) \times(m+1)}$. Hence, by analogy with the previous case, the minimal polynomials of $a$ in $\mathcal{A}$ and of $A_{a}$ in $\mathbb{F}^{(m+1) \times(m+1)}$ are identical; so we observe again that the roots of the minimal polynomial of $a$ in $\mathcal{A}$ are the eigenvalues of $A_{a}$.

Examining the first part of Example 1.5, we note that if $\mathcal{A}$ does not have a unit, then $\varphi$ in (1.4) will still satisfy (1.5), hence $\mathcal{A}_{\varphi}=\left\{A_{a}: a \in \mathcal{A}\right\}$ is a homomorphic image of $\mathcal{A}$. However, since $\mathcal{A}$ lacks a unit, $\varphi$ may fail to be an isomorphism; so contrary to our findings above, the minimal polynomials of an element $a$ in $\mathcal{A}$ and of the corresponding matrix $A_{a}=\varphi(a)$ may differ.

For instance, if

$$
\mathcal{A}=\{\alpha a: \alpha \in \mathbb{F}\}
$$

is a one-dimensional algebra generated by a single element $a \neq 0$ such that $a^{2}=0$, then the minimal polynomial of $a$ in $\mathcal{A}$ is $p_{a}(t)=t^{2}$. On the other hand, $T_{a}$ is the zero transformation on $\mathcal{A}$; so $A_{a}=0$, and the corresponding minimal polynomial is $p_{A_{a}}(t)=t$.

\section{The RADIUS OF AN ELEMENT AND ITS PROPERTIES}

During the entire course of this section we shall assume that the base field $\mathbb{F}$ of our finite-dimensional power-associative algebra $\mathcal{A}$ is either $\mathbb{R}$ or $\mathbb{C}$.

Having established the existence and uniqueness of $p_{a}$, the minimal polynomial of an element $a$ in $\mathcal{A}$, we proceed by defining the radius of $a$ in $\mathcal{A}$ :

$$
r(a)=\max \left\{|\lambda|: \lambda \in \mathbb{C} \text { a root of } p_{a}\right\} .
$$


We note that, unlike the minimal polynomial of an element $a$ in $\mathcal{A}$, the radius $r(a)$ is independent of $\mathcal{A}$ in the sense that if $\mathcal{B}$ is another finite-dimensional powerassociative algebra over $\mathbb{F}$, such that $\mathcal{A}$ is contained in $\mathcal{B}$, then the radii of a in $\mathcal{A}$ and in $\mathcal{B}$ coincide.

Indeed, by Theorem $1.2, p_{a}$ and $q_{a}$, the minimal polynomials of $a$ in $\mathcal{A}$ and in $\mathcal{B}$, are either identical or satisfy $q_{a}(t)=t p_{a}(t)$. Hence, the nonzero roots of $p_{a}$ and $q_{a}$ are equal; so

$$
\max \left\{|\lambda|: \lambda \in \mathbb{C} \text { a root of } p_{a}\right\}=\max \left\{|\lambda|: \lambda \in \mathbb{C} \text { a root of } q_{a}\right\},
$$

and our assertion follows.

In particular, we conclude that if $A$ is a matrix in a subalgebra $\mathcal{A}$ of $\mathbb{F}^{m \times m}$, then the radius of $A$ in $\mathcal{A}$ satisfies

$$
r(A)=\rho(A)
$$

where

$$
\rho(A)=\max \{|\lambda|: \lambda \in \mathbb{C} \text { an eigenvalue of } A\}
$$

is the spectral radius of $A$.

As we shall see in this section, the radius $r$ retains some of the familiar properties of the spectral radius not only for matrices but in the general case as wellproperties that will play a role in Section 3 where we discuss stability of subnorms on subsets of finite-dimensional power-associative algebras.

We begin with the following observations that were noted already in GL3]:

Theorem 2.1 (GL3, Section 2]). Let $\mathcal{A}$ be a finite-dimensional power-associative algebra over $\mathbb{F}$, either $\mathbb{R}$ or $\mathbb{C}$. Then:

(a) The radius $r$ in $(2.1)$ is a nonnegative function on $\mathcal{A}$.

(b) For all $a \in \mathcal{A}$ and $\alpha \in \mathbb{F}$,

$$
r(\alpha a)=|\alpha| r(a) .
$$

(c) For all $a \in \mathcal{A}$ and all positive integers $k$,

$$
r\left(a^{k}\right)=r(a)^{k} .
$$

(d) The radius $r$ vanishes only on nilpotent elements of $\mathcal{A}$.

Proof. We begin with (b). If $\alpha=0$, then there is not much to prove; so suppose $\alpha \neq 0$. Let $p_{a}$ and $p_{\alpha a}$ be the minimal polynomials of $a$ and $\alpha a$ in $\mathcal{A}$, respectively. Then obviously,

$$
p_{\alpha a}(t)=p_{a}\left(\alpha^{-1} t\right)
$$

so the roots of $p_{\alpha a}$ are those of $p_{a}$ multiplied by $\alpha$, and (b) follows.

To prove (c), select $a \in \mathcal{A}$, and let $\mathcal{A}_{a}$ denote the subalgebra of $\mathcal{A}$ generated by $a$. By the remark following the definition of the radius in (2.1), if $b$ is an element in $\mathcal{A}_{a}$, then the radii of $b$ in $\mathcal{A}_{a}$ and in $\mathcal{A}$ coincide. Further, since $\mathcal{A}_{a}$ is associative, we may employ the appropriate isomorphism in Example 1.5 (either $\varphi$ in (1.4) or $\psi$ in (1.6)), by which $\mathcal{A}_{a}$ is algebraically isomorphic to a matrix algebra, such that the roots of the minimal polynomial of $b$ in $\mathcal{A}_{a}$ are the eigenvalues of the corresponding matrix $A_{b}$. Thus,

$$
r(b)=\rho\left(A_{b}\right) \text { for all } b \in \mathcal{A}_{a}
$$


Since $A_{a^{k}}=A_{a}^{k}$, we now get

$$
r\left(a^{k}\right)=\rho\left(A_{a^{k}}\right)=\rho\left(A_{a}^{k}\right)=\rho\left(A_{a}\right)^{k}=r(a)^{k}, \quad k=1,2,3, \ldots,
$$

and $(\mathrm{c})$ is in the bag.

As for (d), we note that $r(a)=0$ if and only if $p_{a}$, the minimal polynomial of $a$ in $\mathcal{A}$, is of the form $p_{a}(t)=t^{n}$ for some positive integer $n$. Hence, $r(a)=0$ if and only if there exists an integer $n$ for which $a^{n}=0$, and the proof is complete.

The rest of this section will be devoted to proving a much deeper property, namely the continuity of $r$ on $\mathcal{A}$.

If $\mathcal{A}$ is associative, then our goal is almost at hand. We appeal to Example 1.5 , and recall that $\mathcal{A}$ is algebraically isomorphic to a subalgebra of $\mathbb{F}^{n \times n}$, where $n=\operatorname{dim} \mathcal{A}$ if $\mathcal{A}$ has a unit and $n=\operatorname{dim} \mathcal{A}+1$ if not, and where the isomorphism associates with each $a \in \mathcal{A}$ a matrix $A_{a} \in \mathbb{F}^{n \times n}$ whose eigenvalues are the roots of the minimal polynomial of $a$ in $\mathcal{A}$. Whence, $r(a)$ coincides with $\rho\left(A_{a}\right)$, the spectral radius of $A_{a}$; and since $\rho$ is a continuous function on $\mathbb{F}^{n \times n}$, we get:

Theorem 2.2 (GL3, Section 2]). Let $\mathcal{A}$ be a finite-dimensional associative algebra over $\mathbb{F}$, either $\mathbb{R}$ or $\mathbb{C}$. Then the radius $r$ is a continuous function on $\mathcal{A}$.

A similar, if more tedious argument holds when we weaken the assumption of associativity and assume that $\mathcal{A}$ is merely alternative, namely, that the subalgebra generated by any two elements in $\mathcal{A}$ is associative.

We begin this argument with the case where $\mathcal{A}$, a finite-dimensional alternative algebra over $\mathbb{F}$, has a unit $e$. Following Example 1.5, let $\operatorname{dim} \mathcal{A}=m$, and consider the regular left representation $T_{a}: \mathcal{A} \rightarrow \mathcal{A}$ in (1.3). Select a basis for $\mathcal{A}$, and for each element $a \in \mathcal{A}$ let $A_{a}$ be the $m \times m$ matrix that represents $T_{a}$ with respect to this basis. It follows that the mapping $\varphi$ in (1.4) satisfies

$$
\varphi(\alpha a+\beta b)=\alpha \varphi(a)+\beta \varphi(b), \quad a, b \in \mathcal{A}, \alpha, \beta \in \mathbb{F} ; \quad \operatorname{Ker} \varphi=\{0\} ;
$$

hence $\mathcal{A}$, regarded as a linear space over $\mathbb{F}$, is isomorphic to $\mathcal{A}_{\varphi}=\left\{A_{a}: a \in \mathcal{A}\right\}$. Further, since $\mathcal{A}$ is alternative, the product $a^{k} x$ is unambiguous for all $a, x \in \mathcal{A}$ and all positive integers $k$. Thus, the linear transformation $T_{a^{k}}$ is well defined and we have $T_{a^{k}}=T_{a}^{k}$; i.e., $A_{a^{k}}=A_{a}^{k}$; or in other words,

$$
\varphi\left(a^{k}\right)=\varphi(a)^{k}, \quad a \in \mathcal{A}, \quad k=1,2,3, \ldots
$$

Moreover, since $T_{e}$ is the identity transformation on $\mathcal{A}$, we have

$$
\varphi(e)=I_{m}
$$

where $I_{m}$ is the identity matrix in $\mathbb{F}^{m \times m}$.

Using (2.3)-(2.5), it is not hard to see that $p_{a}$, the minimal polynomial of $a$ in $\mathcal{A}$, annihilates $A_{a}$, and that in fact, $p_{a}$ is the only monic polynomial of the lowest positive degree that does so. It follows that $p_{a}$ is the minimal polynomial of $A_{a}$ in $\mathbb{F}^{m \times m}$; so the roots of $p_{a}$ are the eigenvalues of $A_{a}$. Consequently, $r(a)=\rho\left(A_{a}\right)$ for all $a \in \mathcal{A}$, and since $\rho$ is continuous on $A_{\varphi}$, so is $r$ on $\mathcal{A}$.

If $\mathcal{A}$ is an $m$-dimensional alternative algebra over $\mathbb{F}$ without a unit, we invoke Example 1.4, and extend $\mathcal{A}$ to the $(m+1)$-dimensional algebra $\mathcal{B}=\mathbb{F} \oplus \mathcal{A}$. Selecting a basis $\left\{b_{1}, \ldots, b_{m+1}\right\}$ for $\mathcal{B}$, we consider $\psi: \mathcal{B} \rightarrow \mathbb{F}^{(m+1) \times(m+1)}$, the mapping in (1.6) that associates with each $a$ in $\mathcal{B}$ the matrix $A_{a}$ which represents the regular left representation $T_{a}: \mathcal{B} \rightarrow \mathcal{B}$ with respect to this basis. As in the previous case where $\mathcal{A}$ had a unit, it follows that $\mathcal{B}$, as a linear space, is isomorphic to $\mathcal{B}_{\psi}=\left\{A_{b}: b \in \mathcal{B}\right\}$; 
so $\mathcal{A}$ is isomorphic to the $m$-dimensional subspace $\left\{A_{\psi}: a \in \mathcal{A}\right\}$ of $\mathcal{B}_{\psi}$, the image of $\mathcal{A}$ under $\psi$. Moreover, we employ the alternativity of $\mathcal{A}$ and, again as in the previous case, we find that the linear isomorphism $\psi$ satisfies

$$
\psi\left(a^{k}\right)=\psi(a)^{k}, \quad a \in \mathcal{A}, \quad k=1,2,3, \ldots
$$

Thus $p_{a}$, the minimal polynomial of $a$ in $\mathcal{A}$, annihilates $A_{a}$.

We claim that $p_{a}$, which is now void of a constant term, coincides with $q_{A_{a}}$, the minimal polynomial of $A_{a}$ in $\mathbb{F}^{(m+1) \times(m+1)}$. Indeed, if $q_{A_{a}}$ has no constant term, then the assertion is easy. On the other hand, if $q_{A_{a}}$ has a constant term, then it is of the form

$$
q_{A_{a}}(t)=\beta_{n} t^{n}+\cdots+\beta_{1} t+\beta_{0}, \quad \beta_{0} \neq 0 .
$$

So (compare (1.2)),

$$
I_{m+1}=-\beta_{0}^{-1}\left(\beta_{n} a^{n}+\cdots+\beta_{1} a\right) \in \mathcal{A}_{\psi},
$$

and it follows that there exists an element $\tilde{e}$ in $\mathcal{A}$ such that the matrix representing $T_{\tilde{e}}$ with respect to our basis is $I_{m+1}$. Therefore, $T_{\tilde{e}}$ is the identity transformation on $\mathcal{B}$. Hence $\tilde{e}$ is a unit in $\mathcal{B}$; so $\mathcal{A}$ has a unit, a contradiction.

Having established the claim stated in the previous paragraph, we conclude, as before, that $r(a)=\rho\left(A_{a}\right)$ for all $a \in \mathcal{A}$; so the continuity of $\rho$ on $\mathcal{A}_{\psi}$ implies that $r$ is continuous on $\mathcal{A}$.

Collecting our findings for the above two cases where $\mathcal{A}$ is alternative (with or without a unit), we may thus register:

Theorem 2.3 (compare GL3, Section 2]). Let $\mathcal{A}$ be a finite-dimensional, alternative algebra over $\mathbb{F}$, either $\mathbb{R}$ or $\mathbb{C}$. Then the radius $r$ is a continuous function on $\mathcal{A}$.

In passing, we recall that, unlike the radius $r$, the coefficients of the minimal polynomial may fail to behave continuously, even when $\mathcal{A}$ is associative. For example, the minimal polynomial of the matrix

$$
A_{\varepsilon}=\left(\begin{array}{ll}
0 & \varepsilon \\
\varepsilon & 0
\end{array}\right), \quad \varepsilon>0
$$

is $p_{A_{\varepsilon}}(t)=t^{2}-\varepsilon^{2}$, whereas for $A_{0}$, the $2 \times 2$ zero matrix, we get $p_{A_{0}}(t)=t$.

To demonstrate the computation of the radius $r$ we offer the following example.

Example 2.1. Viewing the complex numbers,

$$
\mathbb{C}=\{z=\alpha+i \beta: \alpha, \beta \in \mathbb{R}\},
$$

as a 2-dimensional algebra over the reals, it has been noticed in GL3 that the minimal polynomial of $z=\alpha+i \beta \in \mathbb{C}$ is

$$
p_{z}(t)=t^{2}-2 \alpha t+\alpha^{2}+\beta^{2} .
$$

So since the roots of $p_{z}$ are $z$ and $\bar{z}$, it follows that

$$
r(z)=|z| \text {. }
$$

A similar observation (compare, GL1]) can be made by regarding the quaternions,

$$
\mathbb{H}=\{q=\alpha+i \beta+j \gamma+k \delta: \quad \alpha, \beta, \gamma, \delta \in \mathbb{R}\}, \quad i^{2}=j^{2}=k^{2}=i j k=-1,
$$


as a 4-dimensional algebra over $\mathbb{R}$, and recalling the well-known mapping

$$
q \mapsto A_{q} \equiv\left(\begin{array}{cccc}
\alpha & -\beta & -\gamma & -\delta \\
\beta & \alpha & -\delta & \gamma \\
\gamma & \delta & \alpha & -\beta \\
\delta & -\gamma & \beta & \alpha
\end{array}\right), \quad q=\alpha+i \beta+j \gamma+k \delta \in \mathbb{H},
$$

which implies that $\mathbb{H}$ is algebraically isomorphic to the real matrix algebra

$$
A_{4}(\mathbb{R})=\left\{A_{q}: q \in \mathbb{H}\right\} .
$$

Since for each $q=\alpha+i \beta+j \gamma+k \delta \in \mathbb{H}$ the eigenvalues of the corresponding matrix $A_{q}$ are $\alpha \pm i \sqrt{\beta^{2}+\gamma^{2}+\delta^{2}}$ (each with multiplicity 2 ), we appeal to Proposition 1.1 and to $(2.2)$, obtaining

$$
r(q)=r\left(A_{q}\right)=\rho\left(A_{q}\right)=\sqrt{\alpha^{2}+\beta^{2}+\gamma^{2}+\delta^{2}}=|q|, \quad q=\alpha+i \beta+j \gamma+k \delta \in \mathbb{H},
$$

an analogue of (2.6).

The algebraic techniques that led to Theorems 2.2 and 2.3 do not seem to lend themselves to the case where $\mathcal{A}$ is merely power-associative. In this case, our proof that $r$ is continuous is essentially analytic, and we begin with several preliminary remarks.

As usual, a real-valued function

$$
N: \mathcal{A} \rightarrow \mathbb{R}
$$

is a norm on $\mathcal{A}$ if for all $a, b \in \mathcal{A}$ and $\alpha \in \mathbb{F}$,

$$
\begin{aligned}
& N(a)>0, \quad a \neq 0, \\
& N(\alpha a)=|\alpha| N(a), \\
& N(a+b) \leq N(a)+N(b) .
\end{aligned}
$$

We recall that since $\mathcal{A}$ is finite-dimensional, all norms on $\mathcal{A}$ are equivalent, implying a unique topology on $\mathcal{A}$. Thus, selecting a norm $N$, the continuity of $r$ means, of course, that for each $a \in \mathcal{A}$ and $\varepsilon>0$, there exists $\delta>0$, such that if $x \in \mathcal{A}$ satisfies $N(x-a)<\delta$, then $|r(x)-r(a)|<\varepsilon$.

Having selected a norm $N$ on $\mathcal{A}$, we set

$$
\gamma=\max \{N(x y): x, y \in \mathcal{A}, N(x)=N(y)=1\} .
$$

Since $\mathcal{A}$ is finite-dimensional, a simple compactness argument implies that $\gamma$ is a well-defined positive constant satisfying

$$
N(x y) \leq \gamma N(x) N(y), \quad x, y \in \mathcal{A} .
$$

Consequently, using induction, we find that

$$
N\left(x^{k}\right) \leq \gamma^{k-1} N(x)^{k} \quad \text { for all } x \in \mathcal{A}, k=2,3,4, \ldots
$$

The finite-dimensionality of $\mathcal{A}$ also implies that the operations in $\mathcal{A}$ (addition, multiplication, and scalar multiplication) are continuous; so if $\left\{x_{k}\right\}_{k=1}^{\infty}$ and $\left\{y_{k}\right\}_{k=1}^{\infty}$ are sequences in $\mathcal{A}$ that converge to $\tilde{x}$ and $\tilde{y}$ respectively, and if $\left\{\alpha_{k}\right\}_{k=1}^{\infty}$ is a sequence in $\mathbb{F}$ whose limit is $\tilde{\alpha}$, then

$$
\lim _{k \rightarrow \infty}\left(x_{k}+y_{k}\right)=\tilde{x}+\tilde{y}, \quad \lim _{k \rightarrow \infty} x_{k} y_{k}=\tilde{x} \tilde{y}, \quad \lim _{k \rightarrow \infty} \alpha_{k} x_{k}=\tilde{\alpha} \tilde{x} .
$$

A quick way to verify (2.10) is to note that in terms of any linear basis of our finite-dimensional algebra, the convergence of $\left\{x_{k}\right\}_{k=1}^{\infty}$ and $\left\{y_{k}\right\}_{k=1}^{\infty}$ to $\tilde{x}$ and $\tilde{y}$ holds coordinatewise, and that the coordinates of $x_{k}+y_{k}, x_{k} y_{k}$ and $\alpha_{k} x_{k}$ are polynomials (i.e., continuous functions) in the coordinates of $x_{k}$ and $y_{k}$. 
For brevity, we shall refer to the roots of the minimal polynomial of an element $a \in \mathcal{A}$ as the roots of $a$.

Finally, given $\varepsilon>0$ and a point $\zeta_{0} \in \mathbb{C}$, we follow standard terminology and refer to the open set $\left\{\zeta \in \mathbb{C}:\left|\zeta-\zeta_{0}\right|<\varepsilon\right\}$ as the $\varepsilon$-neighborhood of $\zeta_{0}$.

With these preliminaries, we are now ready to prove the following lemma.

Lemma 2.1. Let $\mathcal{A}$ be a finite-dimensional power-associative algebra over $\mathbb{F}$, either $\mathbb{R}$ or $\mathbb{C}$, and let $N$ be a norm on $\mathcal{A}$. Given $a \in \mathcal{A}$ and $\varepsilon>0$, there exists $\delta>0$, such that if $x$ is an element of $\mathcal{A}$ with $N(x-a)<\delta$, then:

(a) Every root $\lambda_{x}$ of $x$ belongs to the $\varepsilon$-neighborhood of some root $\lambda_{a}$ of a.

(b) Every root $\lambda_{a}$ of a belongs to the $\varepsilon$-neighborhood of some root $\lambda_{x}$ of $x$.

Proof 11 Select $x \in \mathcal{A}$, and let $\mathcal{A}_{x}$ be the subalgebra of $\mathcal{A}$ generated by $x$ and by the unit in $\mathcal{A}$ (if $\mathcal{A}$ has one). Then surely, each $y$ in $\mathcal{A}_{x}$ is a polynomial in $x$ with coefficients in $\mathbb{F}$. Let $\lambda_{x}$ be a root of $p_{x}$, the minimal polynomial of $x$ in $\mathcal{A}$, and let

$$
\Gamma_{\lambda_{x}}: \mathcal{A}_{x} \rightarrow \mathbb{C}
$$

be the mapping defined by

$$
\Gamma_{\lambda_{x}}(y)=q_{y}\left(\lambda_{x}\right), \quad y \in \mathcal{A}_{x}
$$

where $q_{y}$ is any polynomial over $\mathbb{F}$ such that $y=q_{y}(x)$. We note that while $q_{y}$ is not unique $\left(y=q_{y}(x)\right.$ implies, for example, $\left.y=q_{y}(x)+p_{x}(x)\right)$, the value of $\Gamma_{\lambda_{x}}(y)$ is uniquely defined. This is so because if $q_{1}$ and $q_{2}$ are two polynomials such that $q_{1}(x)=q_{2}(x)$, then $\left(q_{1}-q_{2}\right)(x)=0$; hence $p_{x}$ divides $q_{1}-q_{2}$, and it follows that $\left(q_{1}-q_{2}\right)\left(\lambda_{x}\right)=0$, i.e., $q_{1}\left(\lambda_{x}\right)=q_{2}\left(\lambda_{x}\right)$.

Take $y_{1}, y_{2} \in \mathcal{A}_{x}$ and $\alpha_{1}, \alpha_{2} \in \mathbb{F}$, and let $q_{1}, q_{2}, q_{3}$, and $q_{4}$ be polynomials over $\mathbb{F}$ such that

$$
y_{1}=q_{1}(x), y_{2}=q_{2}(x), \alpha_{1} y_{1}+\alpha_{2} y_{2}=q_{3}(x), y_{1} y_{2}=q_{4}(x) .
$$

Then,

$$
\left(q_{3}-\alpha_{1} q_{1}-\alpha_{2} q_{2}\right)(x)=0, \quad\left(q_{4}-q_{1} q_{2}\right)(x)=0 ;
$$

so $p_{x}$ divides $q_{3}-\alpha_{1} q_{1}-\alpha_{2} q_{2}$ and $q_{4}-q_{1} q_{2}$, and consequently,

$$
\left(q_{3}-\alpha_{1} q_{1}-\alpha_{2} q_{2}\right)\left(\lambda_{x}\right)=0, \quad\left(q_{4}-q_{1} q_{2}\right)\left(\lambda_{x}\right)=0 .
$$

Hence,

$$
q_{3}\left(\lambda_{x}\right)=\alpha_{1} q_{1}\left(\lambda_{x}\right)+\alpha_{2} q_{2}\left(\lambda_{x}\right), \quad q_{4}\left(\lambda_{x}\right)=q_{1}\left(\lambda_{x}\right) q_{2}\left(\lambda_{x}\right) ;
$$

thus, $\Gamma_{\lambda_{x}}$ is both linear and multiplicative, i.e., for all $y_{1}, y_{2} \in \mathcal{A}_{x}$ and $\alpha_{1}, \alpha_{2} \in \mathbb{F}$,

$$
\Gamma_{\lambda_{x}}\left(\alpha_{1} y_{1}+\alpha_{2} y_{2}\right)=\alpha_{1} \Gamma_{\lambda_{x}}\left(y_{1}\right)+\alpha_{2} \Gamma_{\lambda_{x}}\left(y_{2}\right), \quad \Gamma_{\lambda_{x}}\left(y_{1} y_{2}\right)=\Gamma_{\lambda_{x}}\left(y_{1}\right) \Gamma_{\lambda_{x}}\left(y_{2}\right) .
$$

We claim that

$$
\left|\Gamma_{\lambda_{x}}(y)\right| \leq \gamma N(y) \text { for all } y \in \mathcal{A}_{x}
$$

where $N$ is the given norm on $\mathcal{A}$ and $\gamma$ is the constant defined in (2.8).

To prove (2.12), we fix a linear basis $\left\{b_{1}, \ldots, b_{m}\right\}$ for $\mathcal{A}_{x}$, and let

$$
y=\alpha_{1} b_{1}+\cdots+\alpha_{m} b_{m}
$$

\footnotetext{
${ }^{1}$ The author is grateful to Eliahu Levi for important discussions that greatly facilitated this proof.
} 
be an arbitrary element in $\mathcal{A}_{x}$. Using the linearity of $\Gamma_{\lambda_{x}}$, we obtain

$$
\begin{aligned}
\left|\Gamma_{\lambda_{x}}(y)\right| & =\left|\Gamma_{\lambda_{x}}\left(\alpha_{1} b_{1}+\cdots+\alpha_{m} b_{m}\right)\right| \\
& =\left|\alpha_{1} \Gamma_{\lambda_{x}}\left(b_{1}\right)+\cdots+\alpha_{m} \Gamma_{\lambda_{x}}\left(b_{m}\right)\right| \\
& \leq\left|\alpha_{1}\right|\left|\Gamma_{\lambda_{x}}\left(b_{1}\right)\right|+\cdots+\left|\alpha_{m}\right|\left|\Gamma_{\lambda_{x}}\left(b_{m}\right)\right| .
\end{aligned}
$$

So,

$$
\left|\Gamma_{\lambda_{x}}(y)\right| \leq \kappa_{x} N_{\infty}(y) \text { for all } y \in \mathcal{A}_{x}
$$

where

$$
\kappa_{x}=m \max _{1 \leq j \leq m}\left|\Gamma_{\lambda_{x}}\left(b_{j}\right)\right|
$$

and where

$$
N_{\infty}(y)=\max _{1 \leq j \leq m}\left|\alpha_{j}\right|, \quad y=\alpha_{1} b_{1}+\cdots+\alpha_{m} b_{m} \in \mathcal{A}_{x}
$$

is the sup norm on $\mathcal{A}_{x}$ with respect to our basis.

Next, since all norms on $\mathcal{A}_{x}$ are equivalent, we can exhibit a constant $\kappa_{x}^{\prime}$, such that

$$
N_{\infty}(y) \leq \kappa_{x}^{\prime} N(y), \quad y \in \mathcal{A}_{x}
$$

So by (2.13) and (2.14),

$$
\left|\Gamma_{\lambda_{x}}(y)\right| \leq \kappa_{x}^{\prime \prime} N(y), \quad y \in \mathcal{A}_{x},
$$

where $\kappa_{x}^{\prime \prime}=\kappa_{x} \kappa_{x}^{\prime}$ is a constant depending on $x$.

Finally, we appeal to (2.9), and note that by the multiplicativity of $\Gamma_{\lambda_{x}}$ and by $(2.15)$,

$$
\begin{aligned}
\left|\Gamma_{\lambda_{x}}(y)\right|^{k} & =\left|\Gamma_{\lambda_{x}}\left(y^{k}\right)\right| \\
& \leq \kappa_{x}^{\prime \prime} N\left(y^{k}\right) \leq \kappa_{x}^{\prime \prime} \gamma^{k-1} N(y)^{k} \quad \text { for all } y \in \mathcal{A}_{x} \text { and } k=2,3,4, \ldots
\end{aligned}
$$

Thus,

$$
\left|\Gamma_{\lambda_{x}}(y)\right| \leq \gamma N(y)\left(\frac{\kappa_{x}^{\prime \prime}}{\gamma}\right)^{1 / k} \rightarrow \gamma N(y) \text { as } k \rightarrow \infty
$$

and (2.12) follows.

The inequality in (2.12) has two useful corollaries. First, by taking $y=x$ and $q_{y}(t)=t$, we obtain $\Gamma_{\lambda_{x}}(x)=\lambda_{x}$. Hence, by $(2.12)$,

$$
\left|\lambda_{x}\right| \leq \gamma N(x) \text { for all } x \in \mathcal{A},
$$

i.e., the roots of each $x$ in $\mathcal{A}$ are bounded by $\gamma N(x)$. Second, if $y=q_{y}(x)$ is an element of $\mathcal{A}_{x}$, then by (2.11) and (2.12), the polynomial $q_{y}$ satisfies

$$
\left|q_{y}\left(\lambda_{x}\right)\right| \leq \gamma N\left(q_{y}(x)\right) .
$$

Now suppose part (a) of our lemma is false. Then, for some $a \in \mathcal{A}$ and $\varepsilon>0$, we can find a sequence $\left\{x_{k}\right\}_{k=1}^{\infty}$ in $\mathcal{A}$ with $x_{k} \rightarrow a$, such that each $x_{k}$ has a root $\lambda_{k}$ which does not belong to the $\varepsilon$-neighborhood of any root of $a$.

Since $\left\{x_{k}\right\}_{k=1}^{\infty}$ is a bounded sequence, so is $\left\{\lambda_{k}\right\}_{k=1}^{\infty}$ by (2.16). Thus, passing to an appropriate subsequence if necessary, we may assume that there exists $\tilde{\lambda} \in \mathbb{C}$, such that

$$
\lim _{k \rightarrow \infty} \lambda_{k}=\tilde{\lambda} .
$$


Further, as the $\lambda_{k}$ are not in the $\varepsilon$-neighborhood of any root of $a$, we conclude that $\tilde{\lambda}$ is not a root of $a$. In the next paragraph we will show, however, that $\tilde{\lambda}$ is a root of $a$, a contradiction that will conclude the proof of (a).

Indeed, let $p_{a}$ be the minimal polynomial of $a$ in $\mathcal{A}$. Then by (2.10),

$$
\lim _{k \rightarrow \infty} p_{a}\left(x_{k}\right)=p_{a}(a) \text {. }
$$

Since $p_{a}\left(x_{k}\right)$ is an element of $\mathcal{A}_{x_{k}}$, the subalgebra of $\mathcal{A}$ generated by $x_{k}$ and by the unit of $\mathcal{A}$ (if a unit exists), (2.17) implies

$$
\left|p_{a}(\tilde{\lambda})\right|=\lim _{k \rightarrow \infty}\left|p_{a}\left(\lambda_{k}\right)\right| \leq \gamma \lim _{k \rightarrow \infty} N\left(p_{a}\left(x_{k}\right)\right)=\gamma N\left(p_{a}(a)\right)=0,
$$

and the desired contradiction is at hand.

To prove (b), let

$$
\|p\|_{1}=\left|\alpha_{n}\right|+\cdots+\left|\alpha_{0}\right|, \quad p(t)=\alpha_{n} t^{n}+\cdots+\alpha_{1} t+\alpha_{0} \in \mathbb{C}[t],
$$

denote the $\ell^{1}$ norm on $\mathbb{C}[t]$. Observe that $\|\cdot\|_{1}$ is submultiplicative, i.e.,

$$
\|p q\|_{1} \leq\|p\|_{1}\|q\|_{1}, \quad p, q \in \mathbb{C}[t] .
$$

Given a scalar $\zeta \in \mathbb{C}$ and a polynomial $0 \neq q \in \mathbb{C}[t]$, define the nonnegative function

$$
\Omega_{\zeta}(q)=\frac{|q(\zeta)|}{\|q\|_{1}}
$$

and note that for every $0 \neq \beta \in \mathbb{R}$,

$$
\Omega_{\zeta}(\beta q)=\Omega_{\zeta}(q) .
$$

Clearly,

$$
\|t-\alpha\|_{1}=1+|\alpha|, \quad \alpha \in \mathbb{C} .
$$

So, if

$$
p(t)=\prod_{j=1}^{n}\left(t-\alpha_{j}\right)
$$

is a monic polynomial of positive degree, then by (2.18),

$$
\|p\|_{1} \leq \prod_{j=1}^{n}\left(1+\left|\alpha_{j}\right|\right) .
$$

Thus,

$$
\Omega_{\zeta}(p)=\frac{|p(\zeta)|}{\|p\|_{1}} \geq \frac{|p(\zeta)|}{\prod_{j=1}^{n}\left(1+\left|\alpha_{j}\right|\right)}=\prod_{j=1}^{n} \frac{\left|\zeta-\alpha_{j}\right|}{1+\left|\alpha_{j}\right|} .
$$

Now suppose (b) is false. Then, there exist an element $a \in \mathcal{A}$ with a root $\lambda_{a}$, a positive constant $0<\varepsilon<1$, and a sequence $\left\{x_{k}\right\}_{k=1}^{\infty}$ with $x_{k} \rightarrow a$, such that $\lambda_{a}$ does not belong to the $\varepsilon$-neighborhood of any of the roots of $x_{k}, k=1,2,3, \ldots$.

Denote by $p_{k}$ the minimal polynomial of $x_{k}$ in $\mathcal{A}$, and let the roots of $x_{k}$ be $\lambda_{k, j}$, $j=1, \ldots, n_{k}\left(n_{k}=\operatorname{deg} p_{k} \geq 1\right)$, so that

$$
p_{k}(t)=\prod_{j=1}^{n_{k}}\left(t-\lambda_{k, j}\right) .
$$


Then, by (2.20),

$$
\Omega_{\lambda_{a}}\left(p_{k}\right) \geq \prod_{j=1}^{n_{k}} \frac{\left|\lambda_{a}-\lambda_{k, j}\right|}{1+\left|\lambda_{k, j}\right|}, \quad k=1,2,3, \ldots
$$

Since $\lambda_{a}$ does not belong to the $\varepsilon$-neighborhood of any of the roots of $x_{k}$, we have

$$
\left|\lambda_{a}-\lambda_{k, j}\right| \geq \varepsilon, \quad k=1,2,3, \ldots, \quad j=1, \ldots, n_{k} .
$$

Further, since $\left\{x_{k}\right\}_{k=1}^{\infty}$ is bounded, we use (2.16) to infer that all the $\lambda_{k, j}$ are uniformly bounded; so we can exhibit a constant $\mu>0$ such that

$$
\left|\lambda_{k, j}\right| \leq \mu, \quad k=1,2,3, \ldots, \quad j=1, \ldots, n_{k} .
$$

Thus, by (2.21)-(2.23),

$$
\Omega_{\lambda_{a}}\left(p_{k}\right) \geq\left(\frac{\varepsilon}{1+\mu}\right)^{n_{k}}, \quad k=1,2,3, \ldots
$$

Moreover, setting $m=\operatorname{dim} \mathcal{A}+1$, we note that for every $x \in \mathcal{A}$, the powers

$$
x, x^{2}, \ldots, x^{m}
$$

are linearly dependent. Hence $p_{x}$, the minimal polynomial of $x$ in $\mathcal{A}$, is of degree at most $m$; so by (2.24),

$$
\Omega_{\lambda_{a}}\left(p_{k}\right) \geq\left(\frac{\varepsilon}{1+\mu}\right)^{m}, \quad k=1,2,3, \ldots
$$

Let $\mathbb{C}_{m}[t]$ denote the $(m+1)$-dimensional subspace of $\mathbb{C}[t]$ consisting of all polynomials $p$ with $\operatorname{deg} p \leq m$. Evidently,

$$
p_{x} \in \mathbb{C}_{m}[t] \text { for all } x \in \mathcal{A},
$$

and $\|\cdot\|_{1}$ is a norm on $\mathbb{C}_{m}[t]$.

Since $p_{k} \neq 0$, we put

and observe that

$$
q_{k}=\frac{p_{k}}{\left\|p_{k}\right\|_{1}}
$$

$$
q_{k}\left(x_{k}\right)=0, \quad k=1,2,3, \ldots
$$

As the polynomials $\left\{q_{k}\right\}_{k=1}^{\infty}$ lie on the unit sphere with respect to our $\ell_{1}$ norm on $\mathbb{C}_{m}[t]$, and since this sphere is a compact set, we may assume (passing, if necessary, to a suitable subsequence) that

$$
q_{k} \rightarrow \tilde{q},
$$

where $\tilde{q}$, a member of $\mathbb{C}_{m}[t]$, satisfies $\|\tilde{q}\|_{1}=1$.

Since $\mathbb{C}_{m}[t]$ is finite-dimensional, we note that the convergence in (2.27) holds coefficientwise; that is, for every $j=0, \ldots, m$, the sequence of coefficients of $t^{j}$ in $\left\{q_{k}\right\}_{k=1}^{\infty}$ converges to the coefficient of $t^{j}$ in $\tilde{q}$. Thus, by (2.10), (2.26) and (2.27), we obtain

$$
\tilde{q}(a)=\lim _{k \rightarrow \infty} q_{k}\left(x_{k}\right)=0 .
$$

Consequently, $p_{a}$, the minimal polynomial of $a$ in $\mathcal{A}$, divides $\tilde{q}$. Hence, $\lambda_{a}$ is a root of $\tilde{q}$, so

$$
\Omega_{\lambda_{a}}(\tilde{q})=\frac{\tilde{q}\left(\lambda_{a}\right)}{\|\tilde{q}\|_{1}}=0
$$


On the other hand, by (2.19),

$$
\Omega_{\lambda_{a}}\left(q_{k}\right)=\Omega_{\lambda_{a}}\left(p_{k}\right),
$$

so (2.25) implies

$$
\Omega_{\lambda_{a}}\left(q_{k}\right) \geq\left(\frac{\varepsilon}{1+\mu}\right)^{m}, \quad k=1,2,3, \ldots
$$

Thus, passing to the limit, we get

$$
\Omega_{\lambda_{a}}(\tilde{q}) \geq\left(\frac{\varepsilon}{1+\mu}\right)^{m}
$$

which contradicts (2.28) and so completes the proof.

Our main result in this section is now only a small step away.

Theorem 2.4. Let $\mathcal{A}$ be a finite-dimensional power-associative algebra over $\mathbb{F}$, either $\mathbb{R}$ or $\mathbb{C}$. Then the radius $r$ is a continuous function on $\mathcal{A}$.

Proof. By Lemma 2.1, given $a \in \mathcal{A}$ and $\varepsilon>0$, there exists $\delta>0$, such that if $x$ is an element of $\mathcal{A}$ with $N(x-a)<\delta$, then

$$
r(x) \leq r(a)+\varepsilon \text { and } r(a) \leq r(x)+\varepsilon ;
$$

hence, $|r(x)-r(a)|<\varepsilon$ which forces the desired result.

\section{StABle SUBNORMS}

Throughout this last part of the paper we will follow Section 2 in assuming (often without further mention) that the base field $\mathbb{F}$ of our finite-dimensional powerassociative algebra $\mathcal{A}$ is either $\mathbb{R}$ or $\mathbb{C}$.

Let $\mathcal{S}$, a subset of $\mathcal{A}$, be closed under scalar multiplication (so that $a \in \mathcal{S}$ and $\alpha \in \mathbb{F}$ imply $\alpha a \in \mathcal{S}$ ). Following [GL1], we call a real-valued function

$$
f: \mathcal{S} \rightarrow \mathbb{R}
$$

a subnorm on $\mathcal{S}$ if for all $a \in \mathcal{S}$ and $\alpha \in \mathbb{F}$,

$$
\begin{aligned}
& f(a)>0, \quad a \neq 0, \\
& f(\alpha a)=|\alpha| f(a) .
\end{aligned}
$$

If in addition, $\mathcal{S}$ is closed under raising to powers (i.e., $a \in \mathcal{S}$ implies $a^{k} \in \mathcal{S}$, $k=1,2,3, \ldots)$, then a subnorm $f$ on $\mathcal{S}$ shall be called a submodulus if

$$
f\left(a^{k}\right)=f(a)^{k} \text { for all } a \in \mathcal{S} \text { and } k=1,2,3, \ldots
$$

Finally, if $\mathcal{S}$ is also closed under multiplication, we say that a submodulus $f$ on $\mathcal{S}$ is a modulus if $f$ is multiplicative, i.e.,

$$
f(a b)=f(a) f(b) \text { for all } a, b \in \mathcal{S} .
$$

We recall that if $\mathcal{S}$, a subset of $\mathcal{A}$, is closed under scalar multiplication and under addition, then a real-valued function $N$ is a norm on $\mathcal{S}$ if (2.7) holds for all $a, b \in \mathcal{S}$ and $\alpha \in \mathbb{F}$; thus, in our finite-dimensional context, a norm is a subadditive (hence continuous) subnorm on $\mathcal{S}$.

Examples of subnorms, submoduli and moduli were exhibited in GL1, GL2, GGL, GL3, G]. A selection of these examples is listed below for the reader's convenience. 
Example 3.1. Revisit $\mathbb{C}$, the 2-dimensional algebra of the complex numbers over the reals, and note that for each fixed $p, 0<p \leq \infty$,

$$
|z|_{p}=\left(|\alpha|^{p}+|\beta|^{p}\right)^{1 / p}, \quad z=\alpha+i \beta \in \mathbb{C},
$$

is a continuous subnorm on $\mathbb{C}$. Surely, $|\cdot|_{p}$ is a norm if and only if $1 \leq p \leq \infty$, and a submodulus - in fact, a modulus - only for $p=2$ where we get

$$
|z| \equiv|z|_{2}=\sqrt{\alpha^{2}+\beta^{2}}
$$

Similarly, considering the quaternions, we observe that

$$
|q|_{p}=\left(|\alpha|^{p}+|\beta|^{p}+|\gamma|^{p}+|\delta|^{p}\right)^{1 / p}, \quad q=\alpha+i \beta+j \gamma+k \delta \in \mathbb{H},
$$

is a continuous subnorm for $0<p \leq \infty$, a norm precisely for $1 \leq p \leq \infty$, and a modulus,

$$
|q| \equiv|q|_{2}=\sqrt{\alpha^{2}+\beta^{2}+\gamma^{2}+\delta^{2}}
$$

for $p=2$.

In the same way, we may reflect on the real 8-dimensional alternative algebra of the octonions,

$$
\mathbb{O}=\left\{c=\gamma_{1}+\gamma_{2} e_{2}+\cdots+\gamma_{8} e_{8}: \gamma_{j} \in \mathbb{R}\right\},
$$

with its intricate multiplication rule (e.g., [B]). In analogy with (3.1) and (3.3), we note that

$$
|c|_{p}=\left(\left|\gamma_{1}\right|^{p}+\cdots+\left|\gamma_{8}\right|^{p}\right)^{1 / p}
$$

is a continuous subnorm for $0<p \leq \infty$, a norm if and only if $1 \leq p \leq \infty$, and a modulus,

$$
|c| \equiv|c|_{2}=\sqrt{\gamma_{1}^{2}+\cdots+\gamma_{8}^{2}}
$$

for $p=2$ (a fact that stems from the Eight Square Theorem [D], which implies that $|c d|=|c||d|$ for all $c, d \in \mathbb{O})$.

Example 3.2. Since $\rho$, the spectral radius, vanishes on nonzero nilpotent matrices, it is not a subnorm on $\mathbb{F}^{m \times m}$. It is, however, a subnorm, in fact a continuous submodulus (but usually not a modulus), on any subset of $\mathbb{F}^{m \times m}$ that is void of nonzero nilpotent matrices and closed under scalar multiplication and under raising to powers - for instance, $\mathcal{N}_{m}(\mathbb{F})$, the set of normal $m \times m$ matrices over $\mathbb{F}$.

Example 3.3. Contrary to norms, subnorms and submoduli are often discontinuous. An example of such submoduli is given in [GGL, where the underlying algebra is $\mathbb{F}^{m \times m}$ and the set is $\mathcal{N}_{m}(\mathbb{F})$. Indeed, putting

$$
\tau(A)=\min \{|\lambda|: \lambda \in \mathbb{C} \text { an eigenvalue of } \mathrm{A}\}, \quad A \in \mathcal{N}_{m}(\mathbb{F}),
$$

we observe that

$$
g_{\kappa}(A)= \begin{cases}\rho(A)^{\kappa+1} \tau(A)^{-\kappa}, & \tau(A)>0 \\ \rho(A), & \tau(A)=0\end{cases}
$$

is a submodulus on $\mathcal{N}_{m}(\mathbb{F})$ for every real constant $\kappa$. For $\kappa=0$ we obtain the (continuous) spectral radius. For $\kappa \neq 0$, however, $g_{\kappa}$ is discontinuous, since for the normal matrix

$$
A_{\varepsilon}=\operatorname{diag}(1, \ldots, 1, \varepsilon), \quad \varepsilon>0
$$


one gets

$$
\lim _{\varepsilon \rightarrow 0} g_{\kappa}\left(A_{\varepsilon}\right)= \begin{cases}\infty, & \kappa>0 \\ 0, & \kappa<0\end{cases}
$$

whereas $g_{\kappa}\left(A_{0}\right)=1$.

Example 3.4. We recall that the functional equation

$$
h(s+t)=h(s)+h(t), \quad s, t \in \mathbb{R},
$$

has discontinuous solutions (e.g., HLP, Section 3.20]). It is well known that if $h$ is such a solution, then it is discontinuous everywhere. Further, given a constant $c>0$, one can choose a discontinuous solution $h$ with

$$
h(t+c)=h(t) \text { for all } t \in \mathbb{R},
$$

hence $h$ can be made $c$-periodic. Aided by these facts, it was shown in GL2, Theorem 2.1] that if $f$ is a subnorm on $\mathbb{C}$, the 2-dimensional algebra of the complex numbers over the reals, and if $h$ is a discontinuous $\pi$-periodic solution of (3.8), then:

(a) The real-valued function

$$
g(z)=f(z) e^{h(\arg z)}, \quad z \in \mathbb{C},
$$

is a subnorm on $\mathbb{C}$.

(b) If $f$ is a submodulus or a modulus on $\mathbb{C}$, then so is $g$.

(c) If $f$ is a continuous subnorm on $\mathbb{C}$, then $g$ is discontinuous everywhere in $\mathbb{C}$.

Similar pathological constructions, where the resulting subnorms and submoduli lack any shred of continuity, were obtained in GL2 for the quaternions as well as for $\mathcal{N}_{m}(\mathbb{F})$.

Having recorded Examples 3.1-3.4, we proceed by recalling an elementary result that pertains to continuous subnorms.

Proposition 3.1 (GL1, Lemma 1.2]). Let $\mathcal{S}$, a closed subset of a finite-dimensional power-associative algebra $\mathcal{A}$ over $\mathbb{F}$, either $\mathbb{R}$ or $\mathbb{C}$, be closed under scalar multiplication and under raising to powers. Let $f$ be a continuous subnorm on $\mathcal{S}$ and let $g$ be a continuous submodulus on $\mathcal{S}$. Then,

$$
\lim _{k \rightarrow \infty} f\left(a^{k}\right)^{1 / k}=g(a) \text { for all } a \in \mathcal{S} .
$$

Since the above limit is unique, we immediately get:

Corollary 3.1 (GL1, Corollary 1.1]). Let $\mathcal{A}, \mathcal{S}$ and $g$ be as in Proposition 3.1. Then $g$ is the only continuous submodulus on $\mathcal{S}$.

The definition of submodulus gives rise to another simple, yet basic result:

Theorem 3.1 ([GGL, Proposition 3]). Let $\mathcal{S}$, a subset of a power-associative algebra $\mathcal{A}$ over $\mathbb{F}$, either $\mathbb{R}$ or $\mathbb{C}$, be closed under scalar multiplication and under raising to powers. If $\mathcal{S}$ contains nonzero nilpotent elements, then $\mathcal{S}$ has no submodulus.

This result, which holds for finite- as well as for infinite-dimensional algebras, implies, for instance, that $\mathbb{F}^{m \times m}$ has no submodulus.

Now, let $\mathcal{S}$, a subset of $\mathcal{A}$, be closed under scalar multiplication and under raising to powers. Following GL1, we say that a subnorm $f$ on $\mathcal{S}$ is stable if for some positive constant $\sigma$,

$$
f\left(a^{k}\right) \leq \sigma f(a)^{k} \text { for all } a \in \mathcal{S} \text { and } k=1,2,3, \ldots
$$


We say that $f$ is strongly stable if (3.9) holds for $\sigma=1$. Hence, for example, all submoduli on $\mathcal{S}$ are strongly stable.

With the above definitions, we can now quote the main result in GL1].

Theorem 3.2 (GL1, Theorem 1.1(a)]). Let $\mathcal{S}$, a closed subset of a finite-dimensional power-associative algebra $\mathcal{A}$ over $\mathbb{F}$, either $\mathbb{R}$ or $\mathbb{C}$, be closed under scalar multiplication and under raising to powers. Let $f$ be a continuous subnorm on $\mathcal{S}$, and let $g$ be a continuous submodulus on $\mathcal{S}$. Then $f$ is stable if and only if $f \geq g$ on $\mathcal{S}$.

Appealing to Corollary 3.1, we find, for example, that the modulus functions in (3.2), (3.4) and (3.6) are the only continuous submoduli on $\mathbb{C}, \mathbb{H}$ and $\mathbb{O}$, respectively. Further, by Theorem 3.2 , a continuous subnorm $f$ is stable on $\mathbb{C}, \mathbb{H}$ or $\mathbb{O}$ if and only if $f$ majorizes the corresponding modulus function; so, in particular, the subnorms in (3.1), (3.3) and (3.5) are stable precisely when $0<p \leq 2$.

Another illustration of Corollary 3.1 and Theorem 3.2 is obtained by recalling that $\rho$, the spectral radius, is a continuous submodulus on $\mathcal{N}_{m}(\mathbb{F})$, the set of $m \times m$ normal matrices over $\mathbb{F}$. Since $\mathcal{N}_{m}(\mathbb{F})$ is a closed subset of $\mathbb{F}^{m \times m}$, Corollary 3.1 implies that $\rho$ is the only continuous submodulus on $\mathcal{N}_{m}(\mathbb{F})$, and by Theorem 3.2, a continuous subnorm $f$ is stable on $\mathcal{N}_{m}(\mathbb{F})$ if and only if $f \geq \rho$ there.

We emphasize that the assumption in Corollary 3.1 and in Theorems 3.2 that $\mathcal{S}$ is closed, cannot be dropped. This was established in $\left[\mathrm{G}\right.$ by noting that $G L_{m}(\mathbb{F}) \cup\{0\}$, the union of the general linear group of $m \times m$ invertible matrices over $\mathbb{F}$ and the zero matrix, is not a closed subset of $\mathbb{F}^{m \times m}$, and that both $\rho$ and

$$
\tau(A)=\min \{|\lambda|: \lambda \in \mathbb{C} \text { an eigenvalue of } \mathrm{A}\}
$$

are continuous submoduli on $G L_{m}(\mathbb{F}) \cup\{0\}$. Hence, $\rho$ is not the only continuous submodulus on $G L_{m} \cup(\mathbb{F})\{0\}$, and $\tau$ is stable on $G L_{m}(\mathbb{F}) \cup\{0\}$ without majorizing $\rho$.

We also mention that while a closed set $\mathcal{S}$ has at most one continuous submodulus, $\mathcal{S}$ may have infinitely many discontinuous submoduli, as demonstrated by the action of $g_{\kappa}$ in $(3.7)$ on $\mathcal{N}_{m}(\mathbb{F})$.

With the above survey completed, we are ready to conclude this paper by presenting two new results that hinge on our findings in Section 2 and enhance Corollary 3.1 and Theorem 3.2.

First, we consult Theorems 2.1 and 2.4, which together with Corollary 3.1, yield:

Theorem 3.3. Let $\mathcal{A}$ be a finite-dimensional power-associative algebra over $\mathbb{F}$, either $\mathbb{R}$ or $\mathbb{C}$. Let $\mathcal{S}$, a subset of $\mathcal{A}$, be void of nonzero nilpotents and closed under scalar multiplication and under raising to powers. Then:

(a) (Compare [GL3, Theorem 2.2(b)].) The radius $r$ is a continuous submodulus on $\mathcal{S}$.

(b) If $\mathcal{S}$ is closed, then $r$ is the only continuous submodulus on $\mathcal{S}$.

Now, combining Theorems 3.3(b) and 3.2, we finally get:

Theorem 3.4. Let $\mathcal{S}$, a closed subset of a finite-dimensional power-associative algebra $\mathcal{A}$ over $\mathbb{F}$, either $\mathbb{R}$ or $\mathbb{C}$, be closed under scalar multiplication and under raising to powers. If $\mathcal{S}$ is void of nonzero nilpotents and $f$ is a continuous subnorm on $\mathcal{S}$, then $f$ is stable on $\mathcal{S}$ if and only if $f$ majorizes the radius $r$ on $\mathcal{S}$.

In view of the fundamental role of the radius $r$ in Theorem 3.4, it seems useful to characterize this radius on given algebras - and, in fact, we have done so in several 
familiar cases. For instance, by formula (2.2) and by Example 2.1, we already know that the radius on $\mathbb{F}^{m \times m}$ is the classical spectral radius, and the radii on $\mathbb{C}$ and on $\mathbb{H}$ are the modulus functions in (3.2) and (3.4), respectively. Similarly, since the modulus function in (3.6) is the only continuous submodulus on the octonions $\mathbb{O}$, it follows from Theorem 3.3(b) that this same modulus is the radius on $\mathbb{O}$.

We note that if $\mathcal{A}$ is associative, one can obtain the assertions in Theorems 3.3 and 3.4 by employing Theorem 2.2 instead of Theorem 2.4 (see GL3, Theorem $2.1(\mathrm{~b}, \mathrm{c})]$ and $[\mathrm{G}$, Theorem 3.8]).

Similarly, if $\mathcal{A}$ is alternative, Theorems 3.3 and 3.4 can be obtained by using Theorem 2.3 in place of Theorem 2.4 (see GL3, Theorem 2.3(b,c)] and G, Theorems $3.11])$.

\section{REFERENCES}

[B] J. C. Baez, The octonions, Bull. Amer. Math. Soc. (N. S.) 39 (2002), 145-205. MR1886087 (2003f:17003)

[BM] G. Birkhoff and S. Mac Lane, A Survey of Modern Algebra, Macmillan Publishing Co., New York, 1977. MR0005093(3:99h) (review of original 1941 edition)

[D] L. E. Dickson, On quaternions and their generalization and the history of the Eight Square Theorem, Ann. of Math. 20 (1918-1919), 155-171. MR1502549

[G] M. Goldberg, Stable norms-from theory to applications and back, Linear Algebra Appl. 404 (2005), 223-250. MR2149661 (2006d:15045)

[GGL] M. Goldberg, R. Guralnick and W. A. J. Luxemburg, Stable subnorms II, Linear and Multilinear Algebra 51 (2003), 209-219. MR1976865 (2004c:17001)

[GL1] M. Goldberg and W. A. J. Luxemburg, Stable subnorms, Linear Algebra Appl. 307 (2000), 89-101. MR 1741918 (2001m:15065)

[GL2] M. Goldberg and W. A. J. Luxemburg, Discontinuous subnorms, Linear and Multilinear Algebra 49 (2001), 1-24. MR.1888109(2003a:15026)

[GL3] M. Goldberg and W. A. J. Luxemburg, Stable norms, Pac. J. Math. 215 (2004), 15-27. MR2060492 (2005e:17001)

[HLP] G. H. Hardy, J. E. Littlewood and G. Pólya, Inequalities, Cambridge Univ. Press, Cambridge, 1934.

[R] J. J. Rotman, Advanced Modern Algebra, Pearson Education, Upper Saddle River, New Jersey, 2002. MR2043445 (2005b:00002)

Department of Mathematics, Technion-Israel Institute of Technology, Haifa 32000 , ISRAEL

E-mail address: goldberg@math.technion.ac.il 